\title{
Approaches for improving the performance of filament-type resistive switching memory
}

\author{
LIAN WenTai ${ }^{1,2}$, LONG ShiBing $^{1}$, LÜ HangBing ${ }^{1}$, LIU Qi $^{1,2}$, LI YingTao ${ }^{1}$, ZHANG Sen $^{1}$, \\ WANG Yan ${ }^{1}$, HUO ZongLiang ${ }^{1}$, DAI YueHua ${ }^{2}$, CHEN JunNing ${ }^{2} \&$ LIU Ming ${ }^{1 *}$ \\ ${ }^{1}$ Laboratory of Nano-Fabrication and Novel Device Integration, Institute of Microelectronics, Chinese Academy of Sciences, Beijing 100029, \\ China; \\ ${ }^{2}$ College of Electronics and Technology, Anhui University, Hefei 230039, China
}

Received September 20, 2010; accepted October 12, 2010

Resistive random access memory (RRAM) has received significant research interest because of its promising potential in terms of down-scaling, high density, high speed and low power. However, its endurance, retention and uniformity are still imperfect. In this article, the physical mechanisms of filament-type RRAM and the approaches for improving the switching performance, including doping, process optimization and interface engineering, are introduced.

non-volatile memory, resistive random access memory (RRAM), conductive filament (CF)

Citation: $\quad$ Lian W T, Long S B, Lü H B, et al. Approaches for improving the performance of filament-type resistive switching memory. Chinese Sci Bull, 2011, 56: 461-464, doi: 10.1007/s11434-010-4255-4

Many metal-oxide-metal systems show electrically induced resistive switching (RS) effects and have therefore been proposed as the basis for future non-volatile memories [1,2]. Resistive random access memory (RRAM) will also play an important role in the developing field of logic circuits, field programmable gate arrays and memristors [3]. Depending on where RS occurs, various proposed RS models can simply be classified in to two categories: the interface-type, which can be attributed to the modification of the interface barrier height; and the filament-type, which is cell size independent and only occurs in a localized active area. The filament-type RRAM is thought to be suitable for down-scaling to $20 \mathrm{~nm}$ and below [2]. However, the intrinsic mechanisms are still divergent and robust cycling endurance, data-retention and performance uniformity must be addressed for commercial applications. The key to improving filament-type RRAM is to effectively control and optimize the concentration and profile of inner mobile ions in the binary transition-metal-oxide (TMO) films. This article

*Corresponding author (email: liuming@ime.ac.cn) introduces the approaches for improving the RS performances of filament-type RRAM, including material modifications and interface engineering.

\section{Mechanism of the filament-type RRAM}

In the filament-type RRAM, RS behavior is dominated by the formation and rupture of local conductive paths inside binary TMO films. R. Waser has proposed two models to expound the origins of a filamentary conductive bridge [1].

One model is based on cation migration (electrochemical metallization memory, ECM) [1]. Under applied voltage, the mobile cations coming from the reactive electrode drift and discharge at the counter electrode, leading to the growth of a conductive filament, and finally the cell is turned to the ON-state. With reverse voltage, an electrochemical dissolution of the conductive bridges takes place, resetting the cell into the OFF-state. The validity of the ECM model was convincingly demonstrated by Yang et al. [4].

Another model is based on anion migration (valence 
change memory, VCM) [1]. Usually, the transition metal in the binary TMO has several valences. For example, $\mathrm{Ti}^{4+}$ and $\mathrm{Ti}^{3+}$ (equivalent to an oxygen vacancy) ions coexist in the $\mathrm{TiO}_{2}$ lattice, which indicates local nonstoichiometric $\mathrm{Ti}_{n} \mathrm{O}_{2 n-1}$ (the so-called Magnéli phase) exhibited in the $\mathrm{TiO}_{2}$ film. Under applied voltage, the Magnéli phase filament is extended from anode to cathode because of the generation and movement of oxygen vacancies under a high electric field. When the Magnéli phase filament connects both electrodes, the device transits from the OFF-state to the ON-state attributed to the high conductance of the Magnéli phase. With reverse voltage, the reverse reaction leads to the rupture of filaments, and the device is switched back to the OFF-state. Kwon et al. recently reported the TEM image of the $\mathrm{CF}$ in a $\mathrm{Pt} / \mathrm{TiO}_{2} / \mathrm{Pt}$ device [5]. The results of electron diffraction provide strong evidence for VCM theory.

\section{Performance improvement approaches to filament-type RRAM}

The filament-type RRAM has demonstrated high switching speeds (of the order of $\mathrm{GHz}$ ), large $R_{\mathrm{On}} / R_{\text {Off }}$ ratio $\left(>10^{7}\right)$, low operation voltages $(<1 \mathrm{~V})$ and low switching currents (of the order of nanoamperes) $[4,8,26]$. However, its endurance, retention and uniformity performances have been facing great challenges [1,2]. Many studies have found possible methods for promoting filament-type RRAM $[9,18,23]$. Whatever methods are used, the key is to control and optimize the concentration and profile of inner mobile ions in the binary TMO films. Generally, the improvement methods can be categorized as the following two types: (1) those that modify the functional materials; and (2) those that optimize the interface between the electrode and functional layer.

\subsection{Modification of the functional materials}

The functional layer materials can be modified in the following two ways: (1) doping the oxide materials; and (2) process adjustment in the fabrication of the oxide film.

(i) Doping the functional layer materials. Doping is an effective way to modify the concentration and distribution of impurities, and is widely used in the semiconductor industry. In the field of RRAM, doping impurities is also an important means to improve the performance of the device.
Our group has carried out many studies based on $\mathrm{ZrO}_{2}$ to investigate the effect of doping on the improvement of the performance of the device (as Table 1 shows). Some conclusions can be reached: (1) the initial high electroforming voltage can be eliminated [6-10]; (2) the yield of the devices can be significantly enhanced [8-10]; and (3) the doped devices exhibit more concentrated switching voltage than undoped ones [8-10]. These advantages were also observed in $\mathrm{Cu}$-doped $\mathrm{SiO}_{2}$ [11] and $\mathrm{Al}$-doped $\mathrm{ZnO}$ devices [12].

The intentionally introduced impurities are beneficial for providing sufficient mobile ions in the system. It is well known that the electroforming process is similar to the dielectric soft-breakdown process, in which some defects (such as ions or vacancies) are generated [13]. These defects propagate under a high electrical field and form a local conductive filament between the electrodes. As shown in [6-10], the electroforming processes are all removed, indicating enough defects are generated in $\mathrm{ZrO}_{2}$ film after doping. Because of more uniform and homogeneous defects, high device yields (nearly 100\%) of doped devices can be achieved [8]. Moreover, more stable RS behaviors occur after doping because the impurities introduced play the role of seeds inside the oxide film [10], providing well distributed anchors for the development of filamentary tracks. Thus the RS characteristics of doped devices are more concentrated.

By analyzing the RS characteristics after doping, we can divide dopants into two basic categories. In ECM-based RRAM, doping $\mathrm{Cu}, \mathrm{Ag}$, etc. impurity can increase the concentration of mobile cations [6-9]. While in VCM-based RRAM, more oxygen vacancies will be introduced after doping $\mathrm{Ti}, \mathrm{Li}$, etc, because these impurities have a much stronger ability to absorb oxygen $[10,14,15]$. Based on this characteristic, the Fuji Company reported a Ti-doped NiO RRAM and demonstrated that Ti impurities could produce more oxygen vacancies by SIMS and XPS [14]. However, simply increasing the oxygen vacancies content is not fully effective. How to effectively control the distribution of oxygen vacancy filaments is a key issue to finally improving the RS uniformity. B. Gao et al. indicated doping trivalent elements such as $\mathrm{Al}, \mathrm{La}$ or $\mathrm{Ga}$ into the tetravalent metal oxides such as $\mathrm{HfO}_{2}$ or $\mathrm{ZrO}_{2}$ would lower the forming energy of oxygen vacancies (shown in Table 2), which effectively controls the formation of oxygen vacancy filaments along the doping sites [16].

(ii) Process adjustment during oxide fabrication. As for

Table 1 Some recently reported results of doping $\mathrm{ZrO}_{2}$ film for characteristics improvement

\begin{tabular}{cccccccc}
\hline Device structure & Yield (\%) & Forming process & $V_{\text {set }}(\mathrm{V})$ & $V_{\text {reset }}(\mathrm{V})$ & On/off ratio & Retention (s) & Switching polarity \\
\hline $\mathrm{Au} / \mathrm{Cr}_{\mathrm{ZrO}} / \mathrm{n}^{+} \mathrm{Si}$ & $\sim 50 \%$ & yes & $-2.0--3.5$ & $1.5-3$ & $\sim 10^{3}$ & - & - \\
$\mathrm{Au} / \mathrm{Cr} / \mathrm{ZrO}{ }_{2}: \mathrm{Zr}^{+} / \mathrm{n}^{+} \mathrm{Si}[6]$ & $\sim 90 \%$ & no & 3.2 & -3.2 & $\sim 10^{6}$ & 2500 & bipolar \\
$\mathrm{Au} / \mathrm{Cr} / \mathrm{ZrO}_{2}: \mathrm{Au} / \mathrm{n}^{+} \mathrm{Si}[7]$ & $\sim 90 \%$ & no & $4.5-10$ & $1.5-4$ & $>10^{4}$ & $>10^{6}$ & unipolar \\
$\mathrm{Cu}_{\mathrm{ZrO}} / \mathrm{Pt}$ & $<40 \%$ & yes & $0.5-10$ & $-0.5--1.5$ & $>10^{4}$ & - & bipolar \\
$\mathrm{Cu} / \mathrm{ZrO}_{2}: \mathrm{Cu} / \mathrm{Pt}[8]$ & $\sim 100 \%$ & no & $2.1-3.6$ & $0.8-1.5$ & $\sim 10^{6}$ & $>10^{4}$ & nonpolar \\
$\mathrm{Cu} / \mathrm{ZrO}_{2}: \mathrm{Au} / \mathrm{Pt}[9]$ & $\sim 100 \%$ & no & $2-5$ & $0.5-1.2$ & $>10^{4}$ & $>10^{6}$ & nonpolar \\
$\mathrm{Cu} / \mathrm{ZrO}_{2}: \mathrm{Ti} / \mathrm{Pt}[10]$ & $\sim 100 \%$ & no & $1-4$ & $-0.5--1.5$ & $>10^{4}$ & $>10^{7}$ & bipolar \\
\hline
\end{tabular}


Table 2 Summary of the forming voltage of oxygen vacancy ${ }^{a)}$

\begin{tabular}{cccccc}
\hline & $\begin{array}{c}\text { Undoped } \\
(\mathrm{eV})\end{array}$ & $\begin{array}{c}\text { Ti-doped } \\
(\mathrm{eV})\end{array}$ & $\begin{array}{c}\text { Al-doped } \\
(\mathrm{eV})\end{array}$ & $\begin{array}{c}\text { La-doped } \\
(\mathrm{eV})\end{array}$ & $\begin{array}{c}\text { Ga-doped } \\
(\mathrm{eV})\end{array}$ \\
\hline $\mathrm{HfO}_{2}$ & 6.53 & 6.48 & 4.09 & 3.42 & - \\
$\mathrm{ZrO}_{2}$ & 6.37 & 6.11 & 3.66 & 3.74 & 3.77 \\
\hline
\end{tabular}

a) Data comes from [16].

VCM-based RRAM, the oxygen content and distribution is closely related to the process recipe of the oxide film deposition, such as the oxygen content in the preparation, the film deposition rate and the post-annealing temperature, etc. [17-19].

Different oxygen content (defined as $\mathrm{P}(\%)=\mathrm{P}\left(\mathrm{O}_{2}\right) / \mathrm{P}$ $\left(\mathrm{O}_{2}+\mathrm{Ar}\right)$, $\mathrm{P}$ stands for percentage $)$ in the sputtering atmosphere affects the characteristics of oxide films. When the oxygen content increased from $5 \%$ to $10 \%$, the resistance value of the $\mathrm{NiO}$ film drastically increased, but when the oxygen content increased to $20 \%$, the RS behavior disappeared [17]. This phenomenon demonstrates a moderate amount of Ni vacancies and corresponding cavities can improve switching behavior.

Annealing processes after fabricating the film can improve the film crystallinity, providing more grain boundaries, thus the conductive filament can easily be formed. For some VCM-type RRAM containing oxidizable metal (for example Ti), annealing has great influence on the concentration of oxygen vacancies. As the annealing temperature increases, the oxygen content in the oxide film decreases because of oxygen diffusion and absorption of the oxidizable metal, thus stronger conducting paths with a higher density of oxygen vacancies are created between the two electrodes, leading to the decrease in the forming voltage [19].

\subsection{Interface engineering}

Another important method for controlling the concentration and profile of mobile ions is interface engineering, which mainly includes: (i) selecting a proper electrode; and (ii) inserting a buffer layer between the electrode and oxide film.

(i) Changing the electrode to optimize the interface. The basic memory cell of ECM-based RRAM is a two-terminal element consisting of a solid electrolyte layer sandwiched between an inert and a reactive electrode. The top and bottom electrodes are generally specified, with $\mathrm{Ag}$ or $\mathrm{Cu}$ as the reactive electrode and $\mathrm{Pt}, \mathrm{W}$ or $\mathrm{IrO}$ as the inert electrode [2].

For VCM-based RRAM, different electrodes will cause different behaviors on the interface and device performance. A group of TEM images of the interface engineering are shown in Figure 1. These changed interfaces are composed of oxides of the metal electrode. The reason for the oxidation of the metal electrode is that it has less Gibbs free energy $\left(\Delta G_{0}\right)$ than the metal element in the oxide film. Less $\Delta G_{0}$ means a higher capability to combine with oxygen
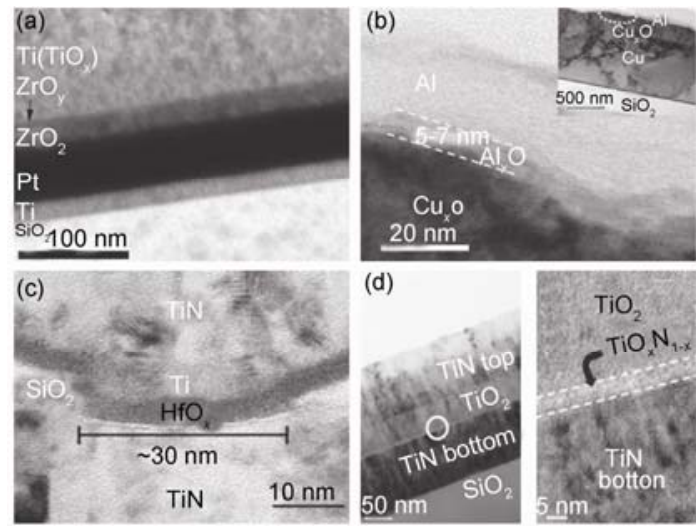

Figure 1 (a) HRTEM image of a thin $\mathrm{ZrO}_{\mathrm{y}}$ interface layer formed between $\mathrm{Ti}\left(\mathrm{TiO}_{x}\right)$ and $\mathrm{ZrO}_{2}$ [21]. (b) Cross-sectional TEM image of the $\mathrm{Al} / \mathrm{Cu}_{x} \mathrm{O} / \mathrm{Cu}$ structure. A clear 5-7-nm-thick interface layer was observed between $\mathrm{Al}$ and $\mathrm{Cu}_{x} \mathrm{O}$ [23]. (c) XTEM image of TiN/TiO $/ \mathrm{HfO}_{x} / \mathrm{TiN}$ device with concave structure [25]. (d) TEM image of $\mathrm{TiN} / \mathrm{TiO}_{2} / \mathrm{TiN}$ cell [24].

[20]. Using such an electrode can produce more oxygen vacancies by absorbing the oxygen in the film, which can make the filament form easily. As shown in Figure 1(a) [21], Ti absorbs oxygen from $\mathrm{ZrO}_{2}$ film, forming $\mathrm{TiO}_{x}$ and $\mathrm{ZrO}_{y}$ on the interface, which makes the oxygen focus in a localized area, thus the conductive filament formation and disruption is localized. Lin et al. have investigated the influence of different types of electrode materials on the interface between the switching layer and the electrode [22]. Commonly, when oxygen-rich oxide materials are used as the switching layer, utilizing $\mathrm{TiN}$, Ti or $\mathrm{Li}$ as TE, which can generate plenty of oxygen vacancies, will greatly improve the RS performance.

In summary, the ECM-type RRAM needs specified electrodes, while for VCM-based RRAM, Ti or TiN electrodes as oxygen reservoirs can provide sufficient nonlattice oxygen ions to recover the oxygen vacancies and form filaments, which will optimize the interface and therefore maintain good switching endurance and retention in the RRAM devices.

(ii) Using a buffer layer to optimize the interface. Introducing a thin buffer layer between the electrode and the functional layer is another effective way to optimize the interface.

As for VCM-based RRAM, the material of the buffer layer is usually oxidizable metal or metal oxide, such as $\mathrm{Ti}$ or $\mathrm{TiO}_{2}$, which is as thin as several nanometers [24-26]. The reason for using such a buffer layer is as follows: for this type of RRAM, the filament formation and rupture are associated with the distribution of movable oxygen ions and oxygen vacancies in the oxide films, and such an oxidizable buffer layer can be considered as the oxygen reservoir to help stabilize the local oxygen migration for the filament formation and rupture (the role is similar to that of the reactive metal electrode discussed previously). According to the RS mechanism of filament-type RRAM discussed above, 
the RS characteristics will be more stable and reliable if the regions where the formation and rupture of the filaments occur can be controlled. By using a thin Ti layer as the reactive buffer layer between the anode and $\mathrm{HfO}_{2}$, excellent memory performances have been demonstrated by Tsai's Group [25]. They also adopted a thin $\mathrm{AlO}_{x}$ buffer layer under the $\mathrm{HfO}_{x}$ to enhance the read disturb immunity of the device [26]. The stacked buffer layer can effectively control the concentration and profile of the mobile oxygen ions, thus leading to a great improvement in the uniform performance of the device.

\section{Conclusions}

The improvement in the performance of RRAM is crucial for practical applications. According to the mechanism of filament-type RRAM, a means of improving the performance of the device should be considered, including ways to control the concentration and profile of inner mobile ions in the functional layer. This article discusses the approach from the following perspectives: modifying the material of the functional layer and optimizing the interface between the electrode and the functional layer. Both doping and process adjustment in fabricating the oxide film can modify the concentration of mobile ions in the functional layer. Changing the electrode or using a buffer layer can achieve the purpose of optimizing the interface between the electrode and the functional layer. Further research is needed to definitively elucidate the 'microscopic' mechanism, which will ultimately provide guidelines for improving RRAM performance.

This work was supported by the National Basic Research Program of China (2010CB934200 and 2008CB925002), the National Natural Science Foundation of China (60825403 and 50972160) and the National HighTech Research \& Development Program of China (2008AA031403 and 2009AA03Z306).

1 Waser R. Resistive non-volatile memory devices. Microelectron Eng, 2009, 86: 1925-1928

2 Kund M, Beitel G, Pinnow C U, et al. Conductive bridging RAM (CBRAM): An emerging non-volatile memory technology scalable to sub $20 \mathrm{~nm}$. IEDM Tech Dig, 2005, 773-776

3 Strukov D B, Snider G S, Stewart D R, et al. The missing memristor found. Nature, 2008, 453: 80-83

4 Yang Y C, Pan F, Liu Q, et al. Fully room-remperature-fabricated nonvolatile resistive memory for ultrafast and high-density memory application. Nano Lett, 2009, 1636-1643

5 Kwon D H, Kim K M, Jang J H, et al. Atomic structure of conducting nanfilaments in $\mathrm{TiO}_{2}$ resistive switching memory. Nat Nanotech, 2010, 5: 148-153
6 Liu Q, Guan W H, Long S B, et al. Resistive switching memory effect of $\mathrm{ZrO}_{2}$ films with $\mathrm{Zr}^{+}$implanted. Appl Phys Lett, 2008, 92: 012117

7 Liu Q, Guan W H, Long S B, et al. Resistive switching of Au-implanted- $\mathrm{ZrO}_{2}$ film for nonvolatile memory application. J Appl Phys, 2008, 104: 114514

8 Guan W H, Liu M, Long S B, et al. On the resistive switching mechanisms of $\mathrm{Cu} / \mathrm{ZrO}_{2}: \mathrm{Cu} / \mathrm{Pt}$. Appl Phys Lett, 2008, 93: 223506

9 Liu Q, Liu M, Long S B, et al. Improvement of resistive switching properties in $\mathrm{ZrO}_{2}$-based ReRAM with implanted metal ions. In: Proc ESSDERC, 2009. 221-224

10 Liu Q, Long S B, Wang W, et al. Improvement of resistive switching properties in $\mathrm{ZrO}_{2}$-based ReRAM with implanted Ti ions. IEEE Electron Device Lett, 2009, 30: 1335-1337

11 Schindler C, Thermadam S C R, Waser R, et al. Bipolar and unipolar resistive switching in $\mathrm{Cu}$-doped $\mathrm{SiO}_{2}$. IEEE Trans Electron Device, 2007, 54: 2762-2768

12 Lee D, Hwang D K, Chang M, et al. Resistance switching of Al doped $\mathrm{ZnO}$ for non volatile memory applications. In: Proc NVSMW, 2006. 86-87

13 Dearnaley G, Stoneham A M, Morgan D V, et al. Electrical phenomenona in amorphous oxide films. Rep Prog Phys, 1970, 33: 1129-1191

14 Tsunoda K, Kinoshita K, Noshiro H, et al. Low power and high speed switching of Ti-doped NiO RRAM under the unipolar voltage source of less than 3 V. IEDM Tech Dig, 2007, 767

15 Jung K, Choi J, Kim Y, et al. Resistance switching characteristics in Li-doped NiO. J Appl Phys, 2008, 103: 034504

16 Gao B, Zhang H W, Yu S, et al. Oxide-Based RRAM: Uniformity improvement using a new material-oriented methodology. VLSI Tech Dig, 2009, 30-31

17 Park J W, Park J W, Jung K, et al. Influence of oxygen content on electrical properties of $\mathrm{NiO}$ films grown by rf reactive sputtering for resistive random-access memory applications. J Vac Sci Technol, 2006, 24: 2205-2208

18 Jung R, Lee M J, Seo S, et al. Decrease in switching voltage fluctuation of $\mathrm{Pt} / \mathrm{NiO}_{x} / \mathrm{Pt}$ structure by process control. Appl Phys Lett, 2007, 91: 022112

19 Lin C Y, Lee D Y, Wang S Y, et al. Effect of thermal treatment on resistive switching characteristics in $\mathrm{Pt} / \mathrm{Ti} / \mathrm{Al}_{2} \mathrm{O}_{3} / \mathrm{Pt}$ devices. Surface Coati Tech, 2008: 628-631

20 Bockris, John O’M R. Modern Electrochemistry. New york: Planum Press, 1998

21 Lin C Y, Wu C Y, Wu C Y, et al. Modified resistive switching behavior of $\mathrm{ZrO}_{2}$ memory films based on the interface layer formed by using Ti top electrode. J Appl Phys, 2007, 102: 094101

22 Lin C Y, Wu C Y, Wu C Y, et al. Effect of top electrode material on resistive switching properties of $\mathrm{ZrO}_{2}$ film memory devices. IEEE Electron Device Lett, 2007, 28: 366-368

$23 \mathrm{Lv} \mathrm{H} \mathrm{B}$, Wang M, Wan H J, et al. Endurance enhancement of $\mathrm{Cu}$-oxide based resistive switching memory with Al top electrode. Appl Phys Lett, 2009, 94: 213502

24 Kwak J S, Do Y H, Bae Y C, et al. Roles of interfacial $\mathrm{TiO}_{x} \mathrm{~N}_{1-x}$ layer and TiN electrode on bipolar resistive switching in $\mathrm{TiN} / \mathrm{TiO}_{2} / \mathrm{TiN}$ frameworks. Appl Phys Lett, 2010, 96: 223502

25 Lee $\mathrm{H} \mathrm{Y,} \mathrm{Chen} \mathrm{P} \mathrm{S,} \mathrm{Wu} \mathrm{T} \mathrm{Y,} \mathrm{et} \mathrm{al.} \mathrm{Low} \mathrm{power} \mathrm{and} \mathrm{high} \mathrm{speed} \mathrm{bipo-}$ lar switching with a thin buffer layer in robust $\mathrm{HfO}_{2}$ Based RRAM. IEDM Tech, 2008, 1-4

26 Chen Y S, Lee H Y, Chen P S, et al. Highly scalable hafnium oxide memory with improvements of resistive distribution and read disturb immunity. IEDM Tech, 2009, 1-4

Open Access This article is distributed under the terms of the Creative Commons Attribution License which permits any use, distribution, and reproduction in any medium, provided the original author(s) and source are credited. 